\title{
CURRENT TRENDS IN INITIAL MANAGEMENT OF HYPOPHARYNGEAL CANCER: THE DECLINING USE OF OPEN SURGERY
}

\author{
Robert P. Takes, MD, PhD, ${ }^{1}$ Primož Strojan, MD, PhD, ${ }^{2}$ Carl E. Silver, $M D,{ }^{3}$ \\ Patrick J. Bradley, MB, BCh, BAO, DCH, MBA, FRCS (Ed, Eng, Ir), FHKCORL, FRCSLT (Hon), FRACS (Hon), ${ }^{4}$ \\ Missak Haigentz, Jr., MD, ${ }^{5}$ Gregory T. Wolf, MD, ${ }^{6}$ Ashok R. Shaha, MD, ${ }^{7}$ \\ Dana M. Hartl, MD, PhD, ${ }^{8,9}$ Jan Olofsson, MD, ${ }^{10}$ Johannes A. Langendijk, MD, PhD, ${ }^{11}$ \\ Alessandra Rinaldo, MD, FRCSEd ad hominem, FRCS (Eng, Ir) ad eundem, FRCSGlasg, ${ }^{12}$ \\ Alfio Ferlito, MD, DLO, DPath, FRCSEd ad hominem, FRCS (Eng, Glasg, Ir) ad eundem, \\ FDSRCS ad eundem, FHKCORL, FRCPath, FASCP, IFCAP ${ }^{12}$ \\ ${ }^{1}$ Department of Otolaryngology-Head and Neck Surgery, Radboud University Nijmegen Medical Center, Nijmegen, \\ The Netherlands \\ ${ }^{2}$ Department of Radiation Oncology, Institute of Oncology, Ljubljana, Slovenia \\ ${ }^{3}$ Departments of Surgery and Otolaryngology-Head and Neck Surgery, Albert Einstein College of Medicine, Montefiore \\ Medical Center, Bronx, NY \\ ${ }^{4}$ Department of Otorhinolaryngology-Head and Neck Surgery, Nottingham University Hospitals, Queens Medical Centre \\ Campus, Nottingham, United Kingdom \\ ${ }^{5}$ Department of Medicine, Division of Oncology, Albert Einstein College of Medicine, Montefiore Medical Center, Bronx, NY \\ ${ }^{6}$ Department of Otolaryngology-Head and Neck Surgery, University of Michigan, Ann Arbor, MI \\ ${ }^{7}$ Head and Neck Service, Memorial Sloan-Kettering Cancer Center, New York, NY \\ ${ }^{8}$ Department of Otolaryngology-Head and Neck Surgery, Institut Gustave Roussy, Villejuif, France \\ ${ }^{9}$ Laboratoire de Phonétique et de Phonologie, Sorbonne Nouvelle, Paris, France \\ ${ }^{10}$ Department of Otolaryngology-Head and Neck Surgery, Haukeland University Hospital, Bergen, Norway \\ ${ }^{11}$ Department of Radiation Oncology, University Medical Center Groningen, University of Groningen, \\ Groningen, The Netherlands \\ ${ }^{12}$ Department of Surgical Sciences, ENT Clinic, University of Udine, Udine, Italy. E-mail: a.ferlito@uniud.it
}

Accepted 12 August 2010

Published online 10 November 2010 in Wiley Online Library (wileyonlinelibrary.com). DOI: 10.1002/hed.21613

\begin{abstract}
Squamous cell carcinoma of the hypopharynx represents a distinct clinical entity. Most patients present with significant comorbidities and advanced-stage disease. The overall survival is relatively poor because of high rates of regional and distant metastasis at presentation or early in the course of the disease. A multidisciplinary approach is crucial in the overall management of these patients to achieve the best results and maintain or improve functional results. Traditionally, operable hypopharyngeal cancer has been treated by total (occasionally partial) laryngectomy and partial or circumferential pharyngectomy, followed by reconstruction and postoperative radiotherapy in most cases.

Efforts to preserve speech and swallowing function in the surgical treatment of hypopharyngeal (and laryngeal) cancer have resulted in a declining use of total laryngopharyngectomy and improved reconstructive efforts, including microvascular free tissue transfer. There are many surgical, as well as nonsurgical, options available for organ and function preservation, which report equally effective tumor control and survival. The
\end{abstract}

Correspondence to: A. Ferlito

This paper was written by members of the International Head and Neck Scientific Group.

() 2010 Wiley Periodicals, Inc selection of appropriate treatment is of crucial importance in the achievement of optimal results for these patients. In this article, several aspects of surgical and nonsurgical approaches in the treatment of hypopharyngeal cancer are discussed. Future studies must be carefully designed within clearly defined populations and use uniform terminology and standardized functional assessment and declare appropriate patient or disease endpoints. These studies should focus on improvement of resultsx, without increasing patient morbidity. In this respect, technical improvements in radiotherapy such as intensity-modulated radiotherapy, advances in supportive care, and incorporation of newer systemic agents such as targeted therapy, are relevant developments. (0 2010 Wiley Periodicals, Inc. Head Neck 34: 270-281, 2012

Keywords: hypopharynx; squamous cell carcinoma; treatment; laryngopharyngectomy; chemoradiotherapy

Squamous cell carcinoma of the hypopharynx is less prevalent than at most other major sites of the head and neck, such as the oral cavity, larynx and oropharynx, and accounts for approximately 3\% to $5 \%$ of all head and neck squamous cell carcinomas (HNSCC). ${ }^{1,2}$ Tumors arising in the hypopharynx have their own 
specific and unique characteristics and considerations regarding treatment. A high proportion of the patients are heavy drinkers of alcohol and have additional significant comorbidities. ${ }^{1}$ Most patients when diagnosed have advanced-stage disease. Approximately $70 \%$ to $85 \%$ of the patients reported in large series have stage III or IV disease at presentation, and the 5-year overall survival rate is reported to be around $15 \%$ to $45 \% .^{1-7}$ The anatomic proximity of the larynx, advanced stage of disease at presentation, and higher rates of regional and distant metastasis portend a worse prognosis compared with other head and neck cancer sites and are factors that require consideration when making treatment decisions.

As reviewed by Gourin and Terris, ${ }^{8}$ the important factors in this dismal prognosis in patients who present with a hypopharyngeal cancer are the high rates of regional and distant metastasis. Approximately $60 \%$ to $80 \%$ of the patients have clinically apparent tumor involved regional lymph nodes, and contralateral occult nodal metastases are present in nearly $40 \%$ of cases. ${ }^{9}$ The number of patients with development of distant metastasis, reported to be between $10 \%$ and $30 \%$ or even higher, is an even more important prognostic factor. Distant metastatic spread has been reported to occur in up to $60 \%$ of hypopharyngeal cancer cases ${ }^{10}$ either at presentation or during follow-up, and is more frequent compared with other HNSCCs. Predominant sites of systemic dissemination are the lung, mediastinal lymph nodes, liver, and bone. These patients are usually treated in the context of various study protocols in which median survival is typically less than a year.

In an epidemiologic study of a series of 595 patients with hypopharyngeal cancer in Ontario, Canada, Hall et $\mathrm{al}^{1}$ reported that almost $50 \%$ of the patients had recurrences, most within 12 months after completion of treatment. Fifty percent of these recurrences involved distant metastasis. Multicentricity is also a common pathologic feature of hypopharyngeal cancers, as well as considerable submucosal spread, and, when treated surgically, the margins of excision frequently demonstrate the presence of tumor. Airway and nutritional issues are difficult problems in patients presenting with advanced hypopharyngeal cancer. Soft tissue extension of the disease involving the constrictor musculature, the soft tissues of the neck, and the parapharyngeal space is quite common.

\section{TREATMENT CHOICES}

Traditionally, laryngopharyngectomy with reconstruction of the pharynx has been the preferred initial treatment modality for hypopharyngeal cancers. In an attempt to limit the morbidity of surgical therapy, nonsurgical treatments have gained popularity. However, treatment with radiotherapy alone is reported to have a worse prognosis compared with combined treatment with surgery and radiotherapy, particularly in stage IV disease., ${ }^{3,6,11}$ The addition of chemotherapy to primary radiotherapy protocols results in out- comes comparable to surgery and postoperative radiotherapy, but with the advantage of larynx preservation in a large number of cases. ${ }^{12}$ However, unlike advanced laryngeal cancers, the question of organ preservation in hypopharyngeal cancer has not been thoroughly evaluated, precluding firm conclusions as to which is the optimal treatment. ${ }^{13,14}$

The terms "organ preservation" and "larynx preservation" in particular are frequently unclearly defined or interpreted. In fact, the terms only mean that the organ has been left anatomically intact and free from surgical intervention but are not indicative of function. Therefore, organ preservation should not be confused with function preservation. With regard to hypopharyngeal cancer, function includes both voice and swallowing and, in a broader sense, quality of life. Function may even be better preserved after removal of the organ, permitting aspiration free deglutition and prosthetic voice than by leaving intact a functionless larynx. Formal studies on this subject are lacking, but physicians involved with this problem are well aware of its existence. In many cases it may be more important to the patient to preserve or restore function rather than to simply preserve an intact but poorly functioning larynx. Stimulated by past experiences and observations, a multidisciplinary international group of experts recently evaluated results from pertinent phase III clinical trials and meta-analyses with the aim of developing guidelines for the conduct of contemporary phase III larynx preservation trials. Emphasis was made in these guidelines on refining the definition of a functional larynx within a context of organ preservation treatment strategies and to determine the best methods for assessment of function. ${ }^{15}$

Moreover, larynx preservation as a treatment strategy, although intended to preserve the organ, includes the option of surgical salvage in cases of persistent or recurrent disease in an effort to obtain optimal patient survival rates comparable to those of primary surgery followed by postoperative (chemo)radiotherapy. Thus organ preserving strategies as a concept for curative treatment are not exclusively nonsurgical.

Neck dissection or irradiation is usually part of the initial treatment of hypopharyngeal cancer because of the high rate of clinically apparent, as well as occult, nodal metastasis. The primary echelon drainage is to the jugular chain (levels II to IV), but the retropharyngeal and level VI nodes are all at risk. ${ }^{16}$ In elective treatment of occult regional metastasis, the high incidence of retropharyngeal and paratracheal metastasis, ${ }^{17}$ as well as the high rate of contralateral metastasis, ${ }^{9}$ should be considered. The low incidence or even absence of nodal metastasis found in sublevel IIB and level I, in particular, may justify the preservation of these levels in the elective treatment of the N0 neck in patients with hypopharyngeal cancer. ${ }^{18-20}$ The several treatment options for hypopharyngeal carcinoma will be discussed. 


\section{PRIMARY SURGERY}

For decades, the treatment protocol that afforded the best oncologic outcomes for hypopharyngeal cancer consisted of radical surgery and postoperative radiotherapy. More recently, the role of initial surgery has been diminished in favor of nonsurgical treatment regimens of radiotherapy combined with platinum-based chemotherapy. However, in early-stage disease, surgery is still a treatment option. Also, in cases of extensive disease, when most patients will already have seriously impaired laryngeal and hypopharyngeal function, surgery with adequate reconstruction, followed by radiotherapy, may have both the best functional and oncologic outcomes.

Three surgical options are available for treatment of hypopharyngeal cancer: "radical" open surgery [total laryngopharyngectomy (TLP) or total laryngectomy with partial pharyngectomy], partial open surgery (partial laryngectomy with pharyngectomy), and partial transoral (or minimally invasive) surgery.

Total Laryngopharyngectomy. Traditionally, lesions that involve more than two thirds of the circumference of the hypopharynx have been treated by radical surgery, consisting of total laryngectomy and circumferential pharyngectomy, including varying amounts of cervical or even thoracic esophagus, followed by radiotherapy in most cases. The 5 -year disease-specific survival rate has been reported to be between $40 \%$ and $50 \% .^{4,21,22}$ Postoperative chemoradiotherapy is reported to further improve tumor control. ${ }^{23}$

The resulting surgical pharyngoesophageal defects require reconstruction and institutional preferences often depend on factors such as the training specialty of the surgical team and the expertise of the available team. ${ }^{24}$ Hypopharyngeal reconstruction has been a major challenge over the years. The deltopectoral flap was the only reconstructive approach before the pectoralis myocutaneous flap became available in the 1970s. The pectoralis myocutaneous flap is a reliable reconstructive method for lesions with minimal extension into the esophagus and has proved useful in severely depleted or elderly patients. ${ }^{25,26}$ This flap, or other myocutaneous flaps are also useful as an onlay patch pharyngoplasty, when a partial pharyngeal defect has been created. Gastric "pull-up" or transposition is generally recommended for tumors that have extended as far as the middle third of the esophagus or where the resected lower margin is deeply placed within the mediastinum, making any safe surgical repair difficult. However, there is a considerable learning curve in performing gastric transposition, which requires careful coordination between the head and neck and thoracic surgeons. More recently the introduction of microvascular and free flap reconstruction has revolutionized the surgical approaches and functional results in hypopharyngeal cancer, both for "patch" or circumferential recon- struction. Microvascular flaps commonly used are jejunum, radial forearm free flap, or anterolateral thigh flap.

Fasciocutaneous free flap, anterolateral thigh flap, and radial forearm free flap reconstructions are currently being used more frequently than the intestinal flaps because of reliability, technical accessibility, and popularity with surgeons. ${ }^{27}$ Functional outcomes have been reported to be better with modern fasciocutaneous free flap reconstruction compared with the traditional jejunal flap, and the donor site morbidity is reported as minimal. ${ }^{27,28}$ However, the morbidity after flap reconstruction is reported to be considerable. In a large surgical series of 153 patients (involving 85 partial and 68 circumferential pharyngectomies, and about $50 \%$ of patients had surgery after previous radiotherapy), fistulas and wound complications were seen in $33 \%$ and $25 \%$ of cases, respectively. The late complication and stricture rates were $26 \%$ and $15 \%$, respectively, and $16 \%$ of patients required permanent feeding through a gastrostomy tube. It was also reported that only $44 \%$ of patients had surgical voice restoration with a tracheoesophageal puncture. ${ }^{29}$

Partial Surgery. More conservative surgical procedures have been used for the treatment of hypopharyngeal cancer. As surgical organ preserving procedures, endoscopic (laser) microsurgery, endoscopic robotic surgery, lateral pharyngectomy, and hemilaryngopharyngectomy are possible endoscopic and external approaches described. However, these procedures can only be considered in selected cases, usually early (T-stage) cancer, and the choice of such limited surgical procedures must recognize that hypopharyngeal cancers have a high predilection to extensive submucosal tumor spread.

Open Procedures. For tumors arising in the upper part of the pyriform sinus or the aryepiglottic fold, a supraglottic hemilaryngopharyngectomy can be used. Although the technique had been used for many decades, one of the first large series of supraglottic laryngectomy and partial pharyngectomy for cancers of the pyriform sinus was published in 1980 by Ogura et al. ${ }^{30}$ In this series of 175 patients treated for carcinoma of the pyriform sinus; 85 underwent partial laryngopharyngectomy (PLP); 57, TLP; and 33, palliative radiation. The actuarial 3 -year survival rate was $59 \%$ for the PLP-treated group, 36\% for the TLP-treated group, and $11 \%$ for the palliation group. The better outcome for the PLP-treated group as compared with the TLP-treated group should be attributed to selection of lower-staged tumors eligible for this treatment.

In 1993, Laccourreye et $\mathrm{al}^{31}$ reported on a series of patients treated between 1964 to 1985 with a supracricoid hemilaryngopharyngectomy (SCHLP) performed on selected pyriform sinus carcinomas classified as T2. Tumors with invasion of the apex of the pyriform sinus, of the postcricoid region, of the 
posterior pharyngeal wall, or with fixation of the true vocal cord were excluded from this study. This technique was aimed at preserving physiologic phonation, respiration, and swallowing while achieving the same local control rate as a pharyngolaryngectomy. Patients were followed up for at least 6 years or until death. Five-year actuarial locoregional control was reported at $95 \%$ with a 5-year disease-specific survival rate of $56 \%$. The 5-year actuarial local recurrence rate was $3.4 \%$. The author considered the SCHLP technique to be a safe method of voice preservation in selected cases of pyriform sinus carcinoma. In 2005, the same group reported on SCHLP performed on 147 patients over a 19-year period. All patients had previously untreated invasive squamous cell carcinoma of the pyriform sinus. $^{32,33}$ Before surgery, almost all $(97 \%)$ of the patients had a cisplatin-based induction chemotherapy regimen. A complete clinical response and a complete histopathologic regression were noted in $22 \%$ and $17 \%$ of patients, respectively. Postoperative radiotherapy was given in approximately $50 \%$ of patients. The 5year actuarial local control estimate was $90 \%$, and the overall laryngeal preservation rate was 91\%. On regression analysis, positive resection margins and invasion of the apex of the pyriform sinus were identified as adverse factors for predicting local recurrence.

In another series of 48 selected cases treated by the same technique of induction chemotherapy followed by surgery, all $\mathrm{T} 1$ or $\mathrm{T} 2$ and arising at the pyriform sinus or aryepiglottic fold, the 5-year survival rate was $47 \% .{ }^{34}$ All patients achieved deglutition without aspiration by the end of the first postoperative month. All but 5 patients received postoperative radiotherapy. Postoperative death or prior radiotherapy was the reason for not undergoing radiotherapy. All patients had ipsilateral (radical) neck dissection. Neck recurrence was $15 \%$, and local recurrence was $2 \%$. Other series report similar results with 5-year overall survival rates around $50 \%$, and disease-specific survival rates of $60 \%$ to $65 \%$ in these selected cases, mostly treated with postoperative radiotherapy and a larynx preservation rate in the order of $80 \% .^{35,36}$

Transoral (Minimally Invasive) Procedures. Transoral laser surgery (TOLS) was initially employed for the resection of laryngeal cancers, ${ }^{37,38}$ but its use was later extended to hypopharyngeal cancer. ${ }^{39,40}$ Zeitels et $\mathrm{al}^{39}$ was one of the first to publish on TOLS for supraglottic and hypopharyngeal cancer. He reported on 45 cases, of which 22 , mostly classified $\mathrm{T} 1$, had TOLS only, and 23 with locally more extensive tumors, but all N0, received additional radiotherapy locally and to the neck. Seven of the 23 cases had positive margins, and $5(26 \%)$ of these failed locoregionally.

Steiner et $\mathrm{al}^{41}$ retrospectively reviewed 129 previously untreated patients with squamous cell carcinoma of the pyriform sinus treated by TOLS. Of these,
24 tumors were staged as pT1, 74 as pT2, 17 as pT3, and 14 as pT4. Overall, $68 \%$ of these patients had nodal metastases. Of all cases, treatment consisted of TOLS and neck dissection surgery alone in $42 \%$, and $58 \%$ also underwent postoperative radiotherapy. The 5-year overall survival rate was $71 \%$ for patients with stage I and II disease, and $47 \%$ for patients with stage III and IV disease. The reported 5-year recurrence-free survival rate for stage I/II and stage III/IV disease was $95 \%$ and $69 \%$, respectively.

Rudert and $\mathrm{Höft}^{42}$ reported a $100 \%$ larynx and pharynx organ preservation rate with a 5-year overall survival rate of $48 \%$ and disease-specific survival rate of $58 \%$ in a series of 29 predominantly $\mathrm{T} 1$ and T2 hypopharyngeal tumors. All but 3 patients underwent postoperative radiotherapy, and all but 4 underwent neck dissection. In this selected group, no symptomatic alteration in swallowing was reported, and the technique was considered suitable for cancers of the posterior hypopharyngeal wall, in particular.

In a series by Vilaseca et $\mathrm{al}^{43}$ of 28 patients treated with TOLS, 21\% (6 patients) had stage II disease, 29 $\%$ (8 patients) stage III, and 50\% (14 patients) stage IV. All patients underwent unilateral or bilateral neck dissection. Of these patients, $43 \%$ had radiotherapy to the neck, and $14 \%$ had radiotherapy locoregionally. Of the 14 patients alive and without disease in the follow-up period, $11(79 \%)$ had their larynx functionally preserved, but 3 (21\%) were gastrostomy dependent.

Kutter et $\mathrm{al}^{44}$ analyzed 55 consecutive patients with pharyngeal or pharyngolaryngeal squamous cell carcinoma $(24=\mathrm{T} 1,28=\mathrm{T} 2,3=\mathrm{T} 3)$ treated with TOLS. Neck dissection was performed in 43 patients. In this group, the 2-year overall survival rate was $78 \%$, and local control was $90 \%$.

Martin et $\mathrm{al}^{45}$ reported on a larger series of 172 patients with hypopharyngeal cancer treated from 1986 to 2003 with TOLS, of whom $15 \%$ had stage I and II disease and $85 \%$ had stage III and IV disease. Of these tumors, $87 \%$ originated in the pyriform sinus. The 5-year overall survival rate was $68 \%$ for stage I and II, $64 \%$ for stage III, and $41 \%$ for stage IV. In this series $52 \%$ underwent postoperative chemoradiation, and $91 \%$ underwent unilateral or bilateral neck dissection. Only 1 patient required laryngectomy for functional reasons, and 6 patients had permanent gastrostomy tubes.

In summary, the oncologic results of TOLS appear comparable with open approaches, with a 5year overall survival rate of around $50 \%$ to $70 \%$ in stage I and II disease and $40 \%$ to $50 \%$ in stage III and IV disease. The disease-specific survival rate with TOLS is on the order of $60 \%$ and is associated with high rates of larynx preservation in these selected cases. However, most patients continue to require postoperative radiotherapy.

Transoral robotic surgery is a minimally invasive surgical technique with the carbon dioxide laser and is currently used for treatment in selected cases and 
selected sites of head and neck cancers. However, the hypopharynx is not optimally accessible for this technique. Published series contain very small numbers of hypopharyngeal cancer cases and mention the aryepiglottic fold or posterior wall as primary tumor sites suitable for this approach. ${ }^{46-48}$

\section{PRIMARY CHEMOTHERAPY OR BIO-RADIOTHERAPY}

As mentioned before, the results of primary definitive radiotherapy followed by salvage surgery when indicated, compared to initial surgery with postoperative radiotherapy, are reported to be inferior in terms of survival, particularly for advanced stage tumors. ${ }^{13,49}$ However, in early-stage hypopharyngeal cancer similar results can be obtained with definitive radiotherapy regimens ${ }^{50}$ with overall survival and diseasespecific survival rates reported to be comparable to those after total laryngectomy or larynx-conserving surgery. ${ }^{51-53}$

In the $1990 \mathrm{~s}$, the first reports on larynx preservation strategies became available. In 1994, the initial report of a phase III study conducted by the Department of Veterans Affairs Laryngeal Cancer Study Group was published. ${ }^{54}$ Patients with stage III-IV laryngeal cancer were randomly assigned to receive 2 cycles of induction chemotherapy with cisplatin and 5-fluorouracil. Those responding (partial or complete) received a third cycle, followed by radiotherapy. Patients not responding clinically to the initial cycles of chemotherapy were offered a total laryngectomy. Survival rates at 2 years appeared to be comparable to surgery followed by radiotherapy (68\% for both treatment groups), and the larynx was preserved in approximately two thirds of the cases. However, patterns of first recurrence differed significantly between the 2 arms, with local recurrences occurring more frequently in the chemotherapy-radiotherapy arm, whereas distant metastases were more common in the surgically treated patients. Overall, there were no significant differences in eventual rate of distant metastases or in cause of death. However, none of these patients were treated for hypopharyngeal cancer.

One of the few randomized phase III trials on hypopharyngeal cancer specifically comparing the results of initial surgical versus nonsurgical treatment was conducted by the European Organization for Research and Treatment of Cancer (EORTC). ${ }^{12}$ Eligible patients with hypopharyngeal or epilaryngeal cancer were randomized between induction PF followed by definitive radiotherapy versus surgery with postoperative radiotherapy. No differences were found in local or regional recurrence and disease-free survival rates at 5 years. The disease-free survival rate at 5 years was $25 \%$ in the induction chemotherapy arm and $27 \%$ in the immediate surgery arm. In this study, the survival rate with a functional larynx in the chemoradiation group after 5 year was $35 \%$, which is similar to other studies. ${ }^{8}$ This larynx preservation rate appears to be inferior compared with that for laryngeal cancer, but it should be emphasized that the 2 endpoints are difficult to compare. First, in the Veterans Affairs Laryngeal Cancer Study Group trial, the larynx preservation rate was reported at 2 years instead of 5 years as in the EORTC study. Moreover, in the EORTC study, the endpoint was referred to as survival with a functional larynx (ie, without local disease, tracheotomy, feeding tube, or gastrostomy).

Recently, the EORTC reported on their second larynx preservation study. ${ }^{55}$ In this study, patients with resectable carcinoma of the larynx (T3-T4, N0-N2) or hypopharynx (T2-T4, N0-N2), were randomly assigned to receive induction chemotherapy followed by radiotherapy in the responders, as described earlier, or an alternating chemoradiation regimen, including a total of 4 cycles of PF (in weeks 1, 4, 7, and 10) alternated with radiotherapy, 20 Gy during the three 2-week intervals between chemotherapy cycles (60 Gy total). The number of laryngeal and hypopharyngeal cancers was well balanced between the 2 arms. Again, the same primary endpoint was used: survival with a functional larynx. The 2 arms showed similar results, as they did with regard to overall survival, progression-free survival, and acute and late toxicity. It should be emphasized that in the alternating chemoradiation arm, the total dose of radiation was reduced to 60 Gy compared with 70 Gy in the sequential arm, and that the overall treatment time of radiation was prolonged as a result of the alternating schedule from 7 weeks to 8 weeks or more. Therefore, it cannot be concluded from this study that sequential chemoradiation provide similar results to conventional concomitant chemoradiation with 70 Gy of radiation with standard fractionation and 3 courses of cisplatin $100 \mathrm{mg} / \mathrm{m}^{2}$ in weeks 1,4 , and 7 .

In 2003 , Forastiere et $\mathrm{al}^{56}$ reported on the results of the RTOG 91-11, a phase III study in which patients with glottic and supraglottic tumors (T2-T4) were randomly assigned to receive induction $\mathrm{PF}$ followed by radiotherapy in responders, concomitant cisplatinbased chemoradiotherapy, or radiotherapy alone. Although overall survival rates were similar in all 3 treatment arms, the highest rate of larynx preservation was observed after concomitant chemoradiation (88\% vs $75 \%$ in the induction chemotherapy arm vs $70 \%$ in the radiotherapy alone arm), which also resulted in superior locoregional control (78\% vs $61 \%$ vs 56\%). But again this trial included laryngeal and not hypopharyngeal cancer. A trial update in 2006 confirmed these results. ${ }^{57}$ It should be noted that in the initial report, salvage total laryngectomy after these organ preservation treatment strategies was associated with acceptable morbidity rates and resulted in excellent locoregional control, although the survival rate at 2 years for patients requiring salvage total laryngectomy was lower than for patients remaining disease-free at the primary site. ${ }^{58}$ However, on the basis of updated results, some concern was raised regarding the high mortality rate 
from unknown causes noted during follow-up, which may have arisen from secondary aspiration, pneumonia, or other unrecognized sequelae of treatment. ${ }^{59}$ Apart from larynx preservation, overall survival only shows modest differences at best between the different regimens for laryngeal cancer and do not seem to significantly differ from "traditional" surgery followed by radiotherapy in the treatment of head and neck cancer in general. ${ }^{60}$

In 2000, Pignon et $\mathrm{al}^{61}$ published the results of individual patient data meta-analysis of 63 randomized trials on locoregional treatment (surgery or radiotherapy) with or without chemotherapy, performed between 1965 and 1993. The study involved 10,741 patients with nonmetastatic HNSCC (MetaAnalysis of Chemotherapy in Head and Neck Cancers). The investigators found an absolute survival benefit of $4 \%$ at 2 and 5 years in favor of the addition of chemotherapy. However, in the group of trials on concomitant chemoradiation, an $8 \%$ absolute benefit at 5 years in the experimental arm was highly significant. The authors concluded at that time that because the analysis showed only a small significant survival benefit in favor of chemotherapy and, in particular, because of the heterogeneity of the results, which required cautious interpretation, "the routine use of chemotherapy is debatable" and that "for larynx preservation, the non-significant negative effect of chemotherapy in the organ preservation strategy indicates that this procedure must remain investigational."

Since that time more studies have been conducted, and the results of the meta-analysis of the updated database of the Meta-Analysis of Chemotherapy in Head and Neck Cancers group with 87 trials conducted between 1965 and 2000, with a total of 16,486 patients, was published recently. ${ }^{62}$ An absolute survival benefit for chemotherapy of $4.5 \%$ at 5 years confirmed previous results, as did the most pronounced benefit observed in concomitant trials $(6.5 \%$ at 5 years). No survival advantage was shown for induction chemotherapy, although this should be interpreted with caution as taxane-based induction chemotherapy trials, which already proved to be superior to the reference PF combination, were not included in meta-analysis. This time the authors concluded that "the benefit of concomitant chemotherapy was confirmed," and results "should be useful to determine standard treatment in this disease." It should be noted that in the initial as in the updated results of the meta-analysis, the subset analysis did not reveal any difference between the different tumor sites. The beneficial effect of the addition of concomitant chemoradiation reference to radiotherapy alone was similar among patients with hypopharyngeal cancer to that observed among patients with other primary tumor sites.

Increasingly intensive treatment regimens have been investigated, not only of concomitant chemora- diotherapy protocols but also sequential approaches consisting of both induction chemotherapy followed by radiotherapy alone or concomitant chemoradiotherapy. The addition of taxanes to the platinumbased induction chemotherapy seems to further improve outcomes, especially for laryngeal preservation. In patients with advanced laryngeal and hypopharyngeal carcinomas, induction chemotherapy consisting of docetaxel, cisplatin, and 5-fluorouracil appeared to be superior to a regimen consisting of $\mathrm{PF}$ in terms of overall response rate (complete and partial responses, $80 \%$ vs $59 \%, p=.002$ ). Larynx preservation could be achieved for a higher proportion of patients in the docetaxel-cisplatin-fluorouracil group compared with the cisplatin-fluorouracil group (at 3 years: $70 \%$ vs $57.5 \%, p=.03$ ), but again without associated overall survival benefit $\left(60 \%\right.$ at 3 years in both arms) ${ }^{63}$

Posner et $\mathrm{al}^{64}$ reported on a similar phase III trial of sequential therapy comparing docetaxel-cisplatin-fluorouracil against cisplatin-fluorouracil induction chemotherapy, followed by chemoradiotherapy with weekly carboplatin. In addition to patients with unresectable oral cavity, oropharyngeal, hypopharyngeal, or laryngeal cancer, patients considered to be candidates for organ preservation were also included (33\% and $35 \%$ of patients in each arm of the trial). Laryngectomy-free survival was significantly greater with docetaxel-cisplatinfluorouracil: at 3 years $52 \%$ vs $32 \%$. Fewer patients treated with docetaxel-cisplatin-fluorouracil underwent surgery ( $22 \%$ vs $42 \%$ ). In this study there was also a significant benefit regarding overall survival in favor of docetaxel-cisplatin-fluorouracil induction chemotherapy.

Another important aspect of nonsurgical approaches is their potential ability to counteract the development of distant metastases. Different patterns of recurrence with fewer initial distant metastases in the induction chemotherapy group compared to the surgery group were observed in The Department of Veterans Affairs' study, ${ }^{54}$ which was confirmed by other authors. ${ }^{65}$ This may be another argument in favor of inclusion of systemic agents in organ preservation treatment protocols. Whereas the concomitant use of irradiation and systemic therapeutics has been proven to successfully improve locoregional disease control and to a lesser extent also distant metastasesfree survival, ${ }^{62}$ the sequential chemoradiation strategies incorporating induction chemotherapy protocols are aimed at diminishing the incidence of distant failures. According to the results of a recent meta-analysis this seems to be the case. ${ }^{62}$

For those patients with M1 disease, prognosis is dismal. Although these patients in particular are directed to take part in experimental clinical protocols exploring new systemic drugs and their combinations, the median survival times are usually less than a year. The results of studies testing a new treatment paradigm combining immunotherapeutic and conventional chemotherapeutics look promising, although tumor sites other than the hypopharynx were included. ${ }^{66}$ 
It may be concluded from the data of these studies that intensifying treatment may increase the rate of larynx preservation but survival benefit has been reported less consistently. ${ }^{59,67,68}$ In addition, interpretation of the (sometimes conflicting) results of chemoradiotherapy trials may be hampered by several factors. Studies often consist of populations with a different mix of both laryngeal and hypopharyngeal cancer and end-points are differently defined or chosen. ${ }^{69}$

\section{TOXICITY OF CHEMORADIATION PROTOCOLS AND QUALITY OF LIFE}

Besides other toxic effects, both acute and late (xerostomia, skin toxicity, cervical fibrosis and lymphedema, ototoxicity), ${ }^{70,71}$ significant swallowing dysfunction is a common finding after intensive chemoradiotherapy. In patients with HNSCC, the rate of symptomatic strictures is estimated to be around $20 \%$, with a hypopharyngeal primary site as a significant predictive factor. ${ }^{72}$ Fixation of the vocal cord, in particular, seems to be a predictor of poor functional outcome, ${ }^{73}$ and even total pharyngeal obstruction may occur. ${ }^{74}$ Impaired swallowing function promotes aspiration, which may lead to pneumonia. Aspiration pneumonia may be an underdocumented and underreported complication of chemoradiotherapy for HNSCC. Future studies should examine whether routine posttherapy videofluoroscopy and swallowing exercise training of patients can reduce this risk. ${ }^{75,76}$

Functional aspects of the results of the Department of Veterans Affairs Laryngeal Cancer Study Group trial related to communication, swallowing and eating were reported by Hillman et al. ${ }^{77}$ From the viewpoint of speech communication, patients treated with induction chemotherapy did better than those with removal of the larynx. For other non-speechrelated measures, there were only a few significant differences between the 2 groups. Only $6 \%$ of patients undergoing total laryngectomy had development of usable esophageal speech, although the proportion of those who remained nonvocal was also small (8\%). When assessing long-term quality of life in surviving patients from the Veterans' study, more favorable results were found in non-surgically treated patients. Better scores in various quality of life instruments appeared to be related to more freedom from pain, better emotional well-being, and lover levels of depression than to preservation of speech function. ${ }^{78}$ Furthermore, Fung et $\mathrm{al}^{79}$ reported on voice and swallowing outcomes of an organ preservation phase II trial for 97 patients with advanced laryngeal cancer. They were treated with a single course of PF induction chemotherapy followed by concurrent chemoradiation when the response was $\geq 50 \%$, or with salvage laryngectomy when response was assessed to be less than $50 \%$. Patients with an intact larynx demonstrated better voice-related quality of life than did patients treated with laryngectomy. Early salvage (immediately after unsuccessful induction chemotherapy) was associated with fewer surgical complications but no difference in voice-related outcome when compared with late laryngectomy (after chemoradiation failure). Although overall swallowing function was good in all patients, those with an intact larynx were more likely to obtain nutrition with oral intake alone without supplements.

Although the above mentioned developments and innovations have led to more intensive treatment approaches aimed at the best possible oncologic results, including laryngeal preservation rate, the morbidity connected to such treatment has increased accordingly. Probably the most important factors contributing to morbidity in these patients is dysphagia and aspiration as a consequence of radiotherapy to the larynx and pharynx. In this respect, relevant structures are the pharyngeal constrictors and supraglottic larynx. ${ }^{80}$ More accurate delineation of target volumes may spare those critical structures. Intensity-modulated radiotherapy (IMRT) offers the possibility of reducing the volumes of these critical structures receiving high doses, ${ }^{81}$ although in hypopharyngeal primaries in particular, growing next to or directly infiltrating structures important for intact swallowing function, such sparing effect of advanced irradiation techniques is less likely. Indeed, a prospective observational study showed that patients treated with IMRT had fewer swallowing problems after completion of radiotherapy or chemoradiation than patients treated with conventional three-dimensional conventional radiotherapy. ${ }^{82}$ Most centers are also trying to spare high-dose radiation to one or more of the major salivary glands with these techniques, which has a beneficial effect on deglutition. ${ }^{83}$

Other recent developments are focused on reducing overlapping toxicity of concomitantly administered systemic agents and irradiation, specifically acute toxicity, lack of compliance and, consequently, late effects affecting function and quality of life. One of these developments is the use of biologic agents as radiosensitizers such as epidermal growth factor receptor inhibitors administered concurrently with either definitive radiotherapy or chemoradiotherapy regimens. ${ }^{84}$ In a phase III randomized trial of radiotherapy and radiotherapy plus weekly doses of cetuximab, in patients with locoregionally advanced HNSCC, the 5-year overall survival rate was significantly improved with the addition of cetuximab, increasing from $36 \%$ to $46 \%$, respectively. ${ }^{85}$ In subgroup analysis, patients with oropharyngeal cancer seemed to benefit more than those with hypopharyngeal cancer, but the study was not powered for this additional analysis. There are no studies on the effectiveness of this treatment regimen in hypopharyngeal cancer in particular. So although potentially promising, possibly in combination with existing chemoradiotherapy regimens, the role of targeted systemic therapies in 
the treatment of hypopharyngeal cancer remains to be established. Moreover, although intended to be less toxic, there may still be considerable side effects, particularly when used in combination with chemoradiotherapy.

Finally, in a study on quality of life of patients with HNSCC, no significant difference was found in overall health-related quality of life between patients treated with surgery followed by radiotherapy and patients treated with chemoradiation. The authors stated that because nonsurgical means of treating head and neck cancer have become more aggressive and surgical techniques have become more focused on function preservation and rehabilitation, the outcome from these 2 approaches is similar in terms of overall health-related quality of life. ${ }^{86}$ In a long-term longitudinal study of quality of life, the presence of a feeding gastrostomy was found to be the variable most closely associated with poor quality of life. ${ }^{87}$ This contrasts many surgeons' perception that the need for long-term tracheostomy or tracheostoma is the most serious functional sequel of treatment. However, the symbolic importance for the patients of keeping an intact larynx should not be underestimated. The French larynx preservation trial on induction chemotherapy conducted in the 1990s had to be closed prematurely because of strong patients' preference for the larynx preservation arm and insufficient accrual into the total laryngectomy arm. ${ }^{88}$

\section{SALVAGE SURGERY}

Although the focus of this article is on initial treatment, salvage surgery could be considered part of planned treatment by nonsurgical approaches. High rates of patient survival in larynx preservation trials are achieved because of effective salvage surgery for locoregional recurrences. The timing and means of surveillance and intervention are still a matter of debate,$^{89,90}$ but are undoubtably of importance. In the initial phase of the chemoradiation era planned neck dissections were the rule. Later it was realized that not all patients needed neck dissection. Conscientious surveillance and timely identification of those patients with resistant or recurrent disease for neck dissection made planned neck dissection obsolete. ${ }^{91}$ Adequate and timely salvage surgery is important to obtain satisfactory locoregional control rates in cases of nonsurgical treatment strategies. 5-year local and regional control rates for salvage pharyngectomy have been reported in $71 \%$ and $70 \%$ of the cases, respectively. ${ }^{92}$ However, less favorable results have also been reported. In a series reported by Relic et al, ${ }^{93}$ only 2 of 20 patients undergoing surgery for histologically proven recurrence of laryngeal or hypopharyngeal cancer after chemoradiotherapy were tumor-free and alive after a mean observation time of 44 months.

Moreover, the rate of complications of surgery after chemoradiation has increased. For example, a high (up to $75 \%$ ) rate of fistulas has been reported, depending on the interval between completion of radiotherapy and salvage surgery, and the dose of radiotherapy. Salvage surgery is associated with longterm stricture rates of $15 \% .^{92,94-96}$

\section{CONCLUSIONS}

Efforts to preserve speech and swallowing function in the treatment of hypopharyngeal (and laryngeal) cancer have resulted in a declining use of TLP. Surgical, as well as nonsurgical, options are available in an attempt to achieve these goals without losing the efficacy of treatment or ultimate tumor control and survival. However, when comparing data on survival, it should be kept in mind that the populations that have been treated with certain modalities are often selected and therefore comparison of results is difficult and should be done with caution (Table 1). ${ }^{4,7,12,21,22,30,31,34-36,41-45,49,51-53}$

With surgical approaches, PLP, as an open procedure or performed endoscopically as a less traumatic minimally invasive procedure, have offered the opportunity of preserving function of the larynx in selected cases of hypopharyngeal cancer. However, only selected cases will be suitable for this approach.

Many patients have benefited from the developments in nonsurgical treatment. A significant number of these patients retain their larynx, which otherwise would have been removed. The treatment is applicable to a wide spectrum of hypopharyngeal cancers, including intermediate-stage disease; only extensive disease with loss of function at presentation could best be treated with TLP as the primary option. However, although increasingly toxic chemoradiation protocols may further improve larynx preservation rates, this could be associated with significantly more pronounced toxicity and worse functional results. Developments aimed at improving results without increasing toxicity and morbidity are focused on technical improvements in radiotherapy, advances in supportive care and the use of new targeted therapy agents other than chemotherapeutics.

In early-stage disease, both surgery and radiotherapy are organ and function preserving treatment options. However, the role of surgery in early-stage hypopharyngeal cancer is less established than for laryngeal cancer. The choice of treatment may depend on patient-related factors like age, comorbidity, ${ }^{97}$ occupation, patient preferences, and tumor-related factors such as size and localization. In advanced disease, most patients will be treated either with TLP followed by (chemo)radiotherapy or up-front chemoradiation. In selected cases partial surgery may be considered, but experience is greater with laryngeal cancer than with hypopharyngeal carcinoma, and favorable results can only be obtained in carefully selected patients and treatment by highly experienced teams. 


\begin{tabular}{|c|c|c|c|}
\hline Treatment & $\begin{array}{l}\text { No. of } \\
\text { patients }\end{array}$ & $\begin{array}{l}\text { Overall survival, } 5 \text { year } \\
\text { (4 year) [3 year] }\{2 \text { year }\}\end{array}$ & $\begin{array}{c}\text { Disease-specific survival, } 5 \text { year } \\
\text { (4 year) [3 year] }\end{array}$ \\
\hline \multicolumn{4}{|l|}{ TLP } \\
\hline Kraus et al ${ }^{4}$ & 132 & $30 \%$ & $41 \%$ \\
\hline \multirow{2}{*}{ Frank et al ${ }^{21}$} & 110 & $18 \% *$ & - \\
\hline & & $48 \%$ & - \\
\hline Bova et al ${ }^{22}$ & 180 & $33 \%$ & $52 \%$ \\
\hline Ogura et al ${ }^{30}$ & 57 & [36\%] & - \\
\hline \multicolumn{4}{|l|}{ Partial surgery } \\
\hline \multicolumn{4}{|l|}{ Open procedures } \\
\hline Ogura et al ${ }^{30}$ & 85 & [59\%] & - \\
\hline Laccourreye et al ${ }^{31}$ & 34 (T2) & - & $56 \%$ \\
\hline \multirow{3}{*}{ Chevalier et $\mathrm{al}^{34}$} & $48(\mathrm{~T} 1 / \mathrm{T} 2)$ & $47 \%$ & - \\
\hline & & $\mathrm{T} 1: 78 \%$ & \\
\hline & & T2:38\% & \\
\hline \multirow[t]{3}{*}{ Makeieff et $\mathrm{al}^{35}$} & 87 (T1/T2) & $60 \%$ & - \\
\hline & & $\mathrm{T} 1: 83 \%$ & \\
\hline & & T2:50\% & \\
\hline Plouin-Gaudon et al ${ }^{36}$ & 34 & $50 \%$ & $65 \%$ \\
\hline \multicolumn{4}{|l|}{ Transoral procedures } \\
\hline \multirow[t]{2}{*}{ Steiner et $\mathrm{al}^{41}$} & 129 & $71 \%$ (stage I/II) & $95 \%$ (stage $\mathrm{I} / \mathrm{II}$ ) \\
\hline & & $47 \%$ (stage III/IV) & $69 \%$ (stage III/IV) \\
\hline Rudert and $\mathrm{Höft}^{42}$ & 29 & $48 \%$ & $58 \%$ \\
\hline Vilaseca et $\mathrm{al}^{43}$ & 28 & $(43 \%)$ & $(59 \%)$ \\
\hline Kutter et al ${ }^{44}$ & 58 & $\{78 \%\}$ & - \\
\hline \multirow[t]{3}{*}{ Martin et $\mathrm{al}^{45}$} & 172 & $68 \%$ (stage $I / I I)$ & $96 \%$ (stage $\mathrm{I} / \mathrm{II}$ ) \\
\hline & & $64 \%$ (stage III) & 86\% (stage III) \\
\hline & & $41 \%$ (stage IV) & $57 \%$ (stage IV) \\
\hline \multicolumn{4}{|l|}{ Radiotherapy } \\
\hline Gupta et $\mathrm{al}^{7}$ & 501 & - & {$[41 \%]$} \\
\hline Godballe et al ${ }^{49}$ & 101 & $16 \%$ & $28 \%$ \\
\hline Yoshimura et al ${ }^{51}$ & 77 (stage I/II) & $47 \%$ & $74 \%$ \\
\hline Nakamura et al ${ }^{52}$ & $115($ stage $\mathrm{I} / \mathrm{II})$ & $66 \%$ & $77 \%$ \\
\hline Rabbani et $\mathrm{al}^{53}$ & 123(stage I/II) & $35 \%$ & $61 \%$ \\
\hline \multicolumn{4}{|l|}{ Chemoradiotherapy } \\
\hline \multirow[t]{2}{*}{ Lefebvre et $\mathrm{al}^{12}$} & 100 & {$[57 \%]$} & {$[43 \%]$} \\
\hline & & & $25 \%$ \\
\hline
\end{tabular}

Abbreviation: TLP, Total laryngopharyngectomy.

${ }^{*}$ Without postoperative radiotherapy.

When comparing nonsurgical and surgical treatment options, not only are traditional oncologic outcome measures important. Functional outcome and quality of life, in particular, are important as well. The latter studies are relatively scarce. Although chemoradiation has gained substantial popularity and seems to be an important treatment option at other sites of the head and neck, in hypopharyngeal cancer this is less obvious, both in terms of oncologic, as well as functional outcome. Lack of studies employing nonsurgical treatments exclusively in hypopharyngeal cancer precludes definitive conclusions.

The success of organ preservation protocols in laryngeal cancer has led to an increase in the use of primary chemoradiation in both laryngeal and extralaryngeal primary sites, despite a paucity of randomized clinical trial data comparing nonsurgical treatment to the "gold standard" of surgery followed by postoperative radiation for unfavorable pathologic features, and an increasing recognition that the high surgical salvage rates enjoyed by patients with laryn- geal cancer do not necessarily translate to extralaryngeal sites. Therefore, despite the declining use of (open) surgery, the role of primary surgery in head and neck cancer should perhaps be reviewed. ${ }^{98}$

Although the emphasis has been shifted to nonsurgical treatments, these strategies still involve surgical procedures. Thus the role of the surgeon in treatment and follow-up remains important, ${ }^{99}$ not only as an option for initial treatment, but also for salvage, tumor surveillance and the diagnosis and treatment of complications and functional impairments.

It is recognized that future studies need to be carefully designed with clearly defined populations and uniformity in terminology and endpoints. ${ }^{15,69}$ Moreover, whereas most larynx preservation trials enroll patients with either laryngeal or hypopharyngeal cancer, it should be realized that these tumors are distinct entities. Results in laryngeal cancer may not directly translate to those in hypopharyngeal cancer. Therefore, specific trials for hypopharyngeal cancer would be needed. 
It is plausible that selection is important in the choice of optimal treatment strategies. For surgical approaches to organ preservation, $\mathrm{T}$ classification, anatomic sublocalization, and extension of the primary tumor are more relevant selection criteria than the overall disease stage. In cases of nonsurgical treatment, volume of the primary tumor may be a predictive factor. ${ }^{100}$ How other factors such as a patient's immune reactivity and the innate biology of an individual tumor relates to prognosis and therapy selection have yet to be explored. Most trials involving nonsurgical treatment strategies report on overall stage rather than $\mathrm{T}$ and $\mathrm{N}$ classification but stage may be a suboptimal criterion for selection of treatment. Small primaries classified as T1 and T2 but with extensive nodal metastases will be staged as IV and must be considered separately from large T4 tumors that are otherwise staged similarly. ${ }^{101} \mathrm{~A}$ small primary tumor might very well be treated by partial surgery followed by postoperative radiotherapy when indicated. More extensive primary tumors will not be suitable for organ preserving surgery and may better be treated with chemoradiotherapy regimens with the intention of organ preservation. Very extensive primary tumors with extensive destruction and functional impairment of the larynx may be best treated with initial laryngopharyngectomy.

Thus, the clues to better oncologic, as well as functional, outcomes are probably to be found in appropriate patient selection. However, the optimal means for selection may be difficult to determine. Until more evidence is available to decide reliably about optimal treatment for the individual patient, each patient should be discussed by knowledgeable multidisciplinary teams weighing the many factors and options that influence an optimal and individualized treatment recommendation.

\section{REFERENCES}

1. Hall SF, Groome PA, Irish J, O'Sullivan B. The natural history of patients with squamous cell carcinoma of the hypopharynx. Laryngoscope 2008;118:1362-1371.

2. Cooper JS, Porter K, Mallin K, et al. National Cancer Database report on cancer of the head and neck: 10-year update. Head Neck 2009;31:748-758.

3. Pingree TF, Davis RK, Reichman O, Derrick L. Treatment of hypopharyngeal carcinoma: a 10 -year review of 1,362 cases. Laryngoscope 1987;97:901-904.

4. Kraus DH, Zelefsky MJ, Brock HA, Huo J, Harrison LB, Shah JP. Combined surgery and radiation therapy for squamous cell carcinoma of the hypopharynx. Otolaryngol Head Neck Surg 1997;116:637-641.

5. Eckel HE, Staar S, Volling P, Sittel C, Damm M, Jungehuelsing M. Surgical treatment for hypopharynx carcinoma: feasibility, mortality, and results. Otolaryngol Head Neck Surg 2001; 124:561-569.

6. Sewnaik A, Hoorweg JJ, Knegt PP, Wieringa MH, van der Beek JM, Kerrebijn JD. Treatment of hypopharyngeal carcinoma: analysis of nationwide study in the Netherlands over a 10 -year period. Clin Otolaryngol 2005;30:52-57.

7. Gupta T, Chopra S, Agarwal JP, et al. Squamous cell carcinoma of the hypopharynx: single-institution outcome analysis of a large cohort of patients treated with primary non-surgical approaches. Acta Oncol 2009;48:541-548.
8. Gourin CG, Terris DJ. Carcinoma of the hypopharynx. Surg Oncol Clin N Am 2004;13:81-98.

9. Buckley JG, MacLennan K. Cervical node metastases in laryngeal and hypopharyngeal cancer: a prospective analysis of prevalence and distribution. Head Neck 2000;22:380-385.

10. Kotwall C, Sako K, Razack MS, Rao U, Bakamjian V, Shedd DP. Metastatic patterns in squamous cell cancer of the head and neck. Am J Surg 1987;154:439-442.

11. Kim S, Wu HG, Heo DS, Kim KH, Sung MW, Park CI. Advanced hypopharyngeal carcinoma treatment results according to treatment modalities. Head Neck 2001;23:713-717.

12. Lefebvre JL, Chevalier D, Luboinski B, Kirkpatrick A, Collette L, Sahmoud T. Larynx preservation in pyriform sinus cancer: preliminary results of a European Organization for Research and Treatment of Cancer phase III trial. EORTC Head and Neck Cancer Cooperative Group. J Natl Cancer Inst 1996;88:890-899.

13. Chen SW, Tsai MH, Yang SN, Liang JA, Shiau AC, Lin FJ. Hypopharyngeal cancer treatment based on definitive radiotherapy: who is suitable for laryngeal preservation? J Laryngol Otol 2008;122:506-512.

14. Tsou YA, Hua JH, Lin MH, Tsai MH. Analysis of prognostic factors of chemoradiation therapy for advanced hypopharyngea cancer-does tumor volume correlate with central necrosis and tumor pathology? ORL J Otorhinolaryngol Relat Spec 2006; 68:206-212.

15. Lefebvre JL, Ang KK; Larynx Preservation Consensus Panel. Larynx preservation clinical trial design: key issues and recommendations--a consensus panel summary. Head Neck 2009;31: 429-441.

16. Ferlito A, Medina JE, Silver CE, Rinaldo A, Paleri V. Elective neck dissection for squamous carcinoma of the head and neck. In: Ferlito A, Robbins KT, Silver CE, editors. Neck dissectionManagement of regional disease in head and neck cancer. San Diego: Plural Publishing Company; 2010. p 179-192.

17. Joo YH, Sun DI, Cho KJ, Cho JH, Kim MS. The impact of paratracheal lymph node metastasis in squamous cell carcinoma of the hypopharynx. Eur Arch Otorhinolaryngol 2010;267:945950.

18. Wiegand S, Esters J, Müller HH, et al. Is it necessary to dissect levels I and IIB in hypopharyngeal cancer? Acta Otolaryngol 2010;130:747-752.

19. Ozer E, Karapinar U, Ryoo C, Agrawal A, Schuller DE. When to address level I lymph nodes in neck dissections? Otolaryngol Head Neck Surg 2010;142:355-358.

20. Kim YH, Koo BS, Lim YC, Lee JS, Kim SH, Choi EC. Lymphatic metastases to level IIb in hypopharyngeal squamous cell carcinoma. Arch Otolaryngol Head Neck Surg 2006;132:1060 1064.

21. Frank JL, Garb JL, Kay S, et al. Postoperative radiotherapy improves survival in squamous cell carcinoma of the hypopharynx. Am J Surg 1994;168:476-480.

22. Bova R, Goh R, Poulson M, Coman WB. Total pharyngolaryngectomy for squamous cell carcinoma of the hypopharynx: a review. Laryngoscope 2005;115:864-869.

23. Bernier J, Cooper JS, Pajak TF, et al. Defining risk levels in locally advanced head and neck cancers: a comparative analysis of concurrent postoperative radiation plus chemotherapy trials of the EORTC (\#22931) and RTOG (\# 9501). Head Neck 2005;27:843-850.

24. Richmon JD, Samji HA, Deschler DG. National laryngopharyngectomy and reconstructive surgery survey. Laryngoscope 2009;119:1472-1478.

25. Cusumano R, Silver C, Brauer R, Strauch B. Pectoralis myocutaneous flap for replacement of cervical esophagus. Head Neck 1989;11:450-456.

26. Lam K, Wei V, Lau V. Avoiding stenosis in the tubed greater pectoral flap in pharyngeal repair. Arch Otolaryngol Head Neck Surg 1987;113:428-431.

27. Richmon JD, Brumund KT. Reconstruction of the hypopharynx: current trends. Curr Opin Otolaryngol Head Neck Surg 2007;15:208-212

28. Robb GL, Lewin JS, Deschler DG, et al. Speech and swallowing outcomes in reconstructions of the pharynx and cervical esophagus. Head Neck 2003;25:232-244.

29. Clark JR, Gilbert R, Irish J, Brown D, Neligan P, Gullane PJ. Morbidity after flap reconstruction of hypopharyngeal defects. Laryngoscope 2006;116:173-181.

30. Ogura JH, Marks JE, Freeman RB. Results of conservation surgery for cancers of the supraglottis and pyriform sinus. Laryngoscope 1980;90:591-600. 
31. Laccourreye O, Mérite-Drancy A, Brasnu D, et al. Supracricoid hemilaryngopharyngectomy in selected pyriform sinus carcinoma staged as T2. Laryngoscope 1993;103:1373-1379.

32. Laccourreye O, Ishoo E, de Mones E, Garcia D, Kania R, Hans S. Supracricoid hemilaryngopharyngectomy in patients with invasive squamous cell carcinoma of the pyriform sinus. Part I: Technique, complications, and long-term functional outcome. Ann Otol Rhinol Laryngol 2005;114:25-34.

33. Kania R, Hans S, Garcia D, Brasnu D, De Mones E, Laccourreye $O$. Supracricoid hemilaryngopharyngectomy in patients with invasive squamous cell carcinoma of the pyriform sinus. Part II: Incidence and consequences of local recurrence Ann Otol Rhinol Laryngol 2005;114:95-104.

34. Chevalier D, Watelet JB, Darras JA, Piquet JJ. Supraglottic hemilaryngopharyngectomy plus radiation for the treatment of early lateral margin and pyriform sinus carcinoma. Head Neck 1997;19:1-5.

35. Makeieff M, Mercante G, Jouzdani E, Garrel R, Crampette L, Guerrier B. Supraglottic hemipharyngolaryngectomy for the treatment of T1 and T2 carcinomas of laryngeal margin and piriform sinus. Head Neck 2004;26:701-705.

36. Plouin-Gaudon I, Lengelé B, Desuter G, et al. Conservation laryngeal surgery for selected pyriform sinus cancer. Eur J Surg Oncol 2004;30:1123-1130.

37. Strong MS, Jako GJ. Laser surgery in the larynx. Early clinical experience with continuous CO2 laser. Ann Otol 1972;81:791798

38. Vaughan CW. Transoral laryngeal surgery using the CO2 laser: laboratory experiments and clinical experience. Laryngoscope 1978;88:1399-1420

39. Zeitels SM, Koufman JA, Davis RK, Vaughan CW. Endoscopic treatment of supraglottic and hypopharynx cancer. Laryngoscope 1994;104:71-78.

40. Steiner W. Therapy of hypopharyngeal cancer. Part III: The concept of minimally invasive therapy of cancers of the upper aerodigestive tract with special reference to hypopharyngeal cancer and trans-oral laser microsurgery. HNO 1994;42:104112. (in German).

41. Steiner W, Ambrosch P, Hess CF, Kron M. Organ preservation by transoral laser microsurgery in piriform sinus carcinoma. Otolaryngol Head Neck Surg 2001;124:58-67.

42. Rudert HH, Höft S. Transoral carbon-dioxide laser resection of hypopharyngeal carcinoma. Eur Arch Otorhinolaryngol 2003; 260:198-206.

43. Vilaseca I, Blanch JL, Bernal-Sprekelsen M, Moragas M. CO2 laser surgery: a larynx preservation alternative for selected hypopharyngeal carcinomas. Head Neck 2004;26:953-959.

44. Kutter J, Lang F, Monnier P, Pasche P. Transoral laser surgery for pharyngeal and pharyngolaryngeal carcinomas. Arch Otolaryngol Head Neck Surg 2007;133:139-144.

45. Martin A, Jäckel MC, Christiansen H, Mahmoodzada M, Kron M, Steiner W. Organ preserving transoral laser microsurgery for cancer of the hypopharynx. Laryngoscope 2008;118:398-402.

46. Park YM, Lee WJ, Lee JG, et al. Transoral robotic surgery (TORS) in laryngeal and hypopharyngeal cancer. J Laparoendosc Adv Surg Tech A 2009;19:361-368.

47. Genden EM, Desai S, Sung CK. Transoral robotic surgery for the management of head and neck cancer: a preliminary experience. Head Neck 2009;31:283-289.

48. Boudreaux BA, Rosenthal EL, Magnuson JS, et al. Robotassisted surgery for upper aerodigestive tract neoplasms. Arch Otolaryngol Head Neck Surg 2009;135:397-401.

49. Godballe C, Jørgensen K, Hansen O, Bastholt L. Hypopharyngeal cancer: results of treatment based on radiation therapy and salvage surgery. Laryngoscope 2002;112:834-838.

50. Foote RL. Radiotherapy alone for early-stage squamous cell carcinoma of the larynx and hypopharynx. Int J Radiat Oncol Biol Phys 2007;69:S31-S36.

51. Yoshimura RI, Kagami Y, Ito Y, et al. Outcomes in patients with early-stage hypopharyngeal cancer treated with radiotherapy. Int J Radiat Oncol Biol Phys 2010;77: 1017-1023.

52. Nakamura K, Shioyama Y, Kawashima M, et al. Multi-institutional analysis of early squamous cell carcinoma of the hypopharynx treated with radical radiotherapy. Int J Radiat Oncol Biol Phys 2006;65:1045-1050.

53. Rabbani A, Amdur R, Mancuso A, et al. Definitive radiotherapy for T1-T2 squamous cell carcinoma of pyriform sinus. Int $\mathrm{J}$ Radiat Oncol Biol Phys 2008;72:351-355.

54. The Department of Veterans Affairs Laryngeal Cancer Study Group. Induction chemotherapy plus radiation compared with surgery plus radiation in patients with advanced laryngeal cancer. N Engl J Med 1991;324:1685-1690.

55. Lefebvre JL, Rolland F, Tesselaar M, et al. Phase 3 randomized trial on larynx preservation comparing sequential vs alternating chemotherapy and radiotherapy. J Natl Cancer Inst 2009; 101:142-152.

56. Forastiere AA, Goepfert H, Maor M, et al. Concurrent chemotherapy and radiotherapy for organ preservation in advanced laryngeal cancer. N Engl J Med 2003;349:2091-2098.

57. Forastiere A, Maor M, Weber R, et al. Long-term results of Intergroup RTOG 91-11 phase III trial to preserve the larynx induction cisplatin/5FU and radiation therapy versus concurrent cisplatin and radiation therapy versus radiation therapy. Proc Am Soc Clin Oncol 2006;24:abstr 5517.

58. Weber RS, Berkey BA, Forastiere A, et al. Outcome of salvage total laryngectomy following organ preservation therapy. The Radiation Therapy Oncology Group Trial 91-11. Arch Otolaryngol Head Neck Surg 2003;129:44-49.

59. Olsen KD. Reexamining the treatment of advanced laryngeal cancer. Head Neck 2010;32:1-7.

60. Soo KC, Tan EH, Wee J, et al. Surgery and adjuvant radiotherapy vs concurrent chemoradiotherapy in stage III/IV nonmetastatic squamous cell head and neck cancer: a randomised comparison. Br J Cancer 2005;93:279-286.

61. Pignon JP, Bourhis J, Domenge C, Designé L. Chemotherapy added to locoregional treatment for head and neck squamouscell carcinoma: three meta-analyses of updated individual data. MACH-NC Collaborative Group. Meta-Analysis of Chemotherapy on Head and Neck Cancer. Lancet 2000;355:949-955.

62. Pignon JP, le Maître A, Maillard E, Bourhis J; MACH-NC Collaborative Group. Meta-analysis of chemotherapy in head and neck cancer (MACH-NC): an update on 93 randomized trials and 17,346 patients. Radiother Oncol 2009;92:4-14.

63. Pointreau Y, Garaud P, Chapet S, et al. Randomized trial of induction chemotherapy with cisplatin and 5-fluorouracil with or without docetaxel for larynx preservation. J Natl Cancer Inst 2009;101:498-506.

64. Posner MR, Norris CM, Wirth LJ, et al. Sequential therapy for the locally advanced larynx and hypopharynx cancer subgroup in TAX 324: survival, surgery, and organ preservation. Ann Oncol 2009;20:921-927.

65. Paccagnella A, Orlando A, Marchiori C, et al. Phase III trial of initial chemotherapy in stage III or IV head and neck cancers: a study by the Gruppo di Studio sui Tumori della Testa e del Collo. J Natl Cancer Inst 1994;86:265-272.

66. Vermorken JB, Mesia R, Rivera F, et al. Platinum-based chemotherapy plus cetuximab in head and neck cancer. N Engl J Med 2008;359:1116-1127.

67. Forastiere AA. Larynx preservation and survival trends: should there be concern? Head Neck 2010;32:14-17.

68. Hoffman HT, Porter K, Karnell LH, et al. Laryngeal cancer in the United States: changes in demographics, patterns of care, and survival. Laryngoscope 2006;116:1-13.

69. Forastiere AA, Trotti AM. Searching for less toxic larynx preservation: a need for common definitions and metrics. J Natl Cancer Inst 2009;101:129-131.

70. Rades D, Fehlauer F, Sheikh-Sarraf M, et al. Toxicity of two cisplatin-based radiochemotherapy regimens for the treatment of patients with stage III/IV head and neck cancer. Head Neck 2008;30:235-241.

71. Zuur CL, Simis YJ, Lansdaal PE, et al. Risk factors of ototoxicity after cisplatin-based chemo-irradiation in patients with locally advanced head-and-neck cancer: a multivariate analysis. Int J Radiat Oncol Biol Phys 2007;68:1320-1325.

72. Lee WT, Akst LM, Adelstein DJ, et al. Risk factors for hypopharyngeal/upper esophageal stricture formation after concurrent chemoradiation. Head Neck 2006;28:808-812.

73. Staton J, Robbins KT, Newman L, Samant S, Sebelik M, Vieira F. Factors predictive of poor functional outcome after chemoradiation for advanced laryngeal cancer. Otolaryngol Head Neck Surg 2002;127:43-47.

74. Franzmann EJ, Lundy DS, Abitbol AA, Goodwin WJ. Complete hypopharyngeal obstruction by mucosal adhesions: a complication of intensive chemoradiation for advanced head and neck cancer. Head Neck 2006;28:663-670.

75. Rosenthal DI, Lewin JS, Eisbruch A. Prevention and treatment of dysphagia and aspiration after chemoradiation for head and neck cancer. J Clin Oncol 2006;24:2636-2643.

76. Gluck I, Feng FY, Lyden T, et al. Evaluating and reporting dysphagia in trials of chemoirradiation for head-and-neck cancer. Int J Radiat Oncol Biol Phys 2010;77:727-33 
77. Hillman RE, Walsh MJ, Wolf GT, Fisher SG, Hong WK. Functional outcomes following treatment for advanced laryngeal cancer. Part I-voice preservation in advanced laryngeal cancer. Part II-laryngectomy rehabilitation: the state of the art in the VA System. Research Speech-Language Pathologists. Department of Veterans Affairs Laryngeal Cancer Study Group. Ann Otol Rinol Laryngol Suppl 1998;172:1-27.

78. Terrell JE, Fisher SG, Wolf GT. Long-term quality of life after treatment of laryngeal cancer. The Veterans Affairs Laryngeal Cancer Study Group. Arch Otolaryngol Head Neck Surg 1998;124:964-971.

79. Fung K, Lyden TH, Lee J, et al. Voice and swallowing outcomes of an organ-preservation trial for advanced laryngeal cancer. Int J Radiat Oncol Biol Phys 2005;63:1395-1399.

80. Caglar HB, Tishler RB, Othus M, et al. Dose to larynx predicts for swallowing complications after intensity-modulated radiotherapy. Int J Radiat Oncol Biol Phys 2008;72:1110-1118.

81. Feng FY, Kim HM, Lyden TH, et al. Intensity-modulated radiotherapy of head and neck cancer aiming to reduce dysphagia: early dose-effect relationships for the swallowing structures. Int J Radiat Oncol Biol Phys 2007;68:1289-1298.

82. Vergeer MR, Doornaert PA, Rietveld DH, Leemans CR, Slotman $\mathrm{BJ}$, Langendijk JA. Intensity-modulated radiotherapy reduces radiation-induced morbidity and improves health-related quality of life: results of a nonrandomized prospective study using a standardized follow-up program. Int $J$ Radiat Oncol Biol Phys 2009;74:1-8.

83. Eisbruch A, Ship JA, Dawson LA, et al. Salivary gland sparing and improved target irradiation by conformal and intensity modulated irradiation of head and neck cancer. World J Surg 2003;27:832-837.

84. Marur S, Forastiere AA. Challenges of integrating chemotherapy and targeted therapy with radiation in locally advanced head and neck squamous cell cancer. Curr Opin Oncol 2010;22:206-211.

85. Bonner JA, Harari PM, Giralt J, et al. Radiotherapy plus cetuximab for locoregionally advanced head and neck cancer: 5-year survival data from a phase 3 randomised trial, and relation between cetuximab-induced rash and survival. Lancet Oncol 2010;11:21-28.

86. El-Deiry M, Funk GF, Nalwa S, et al. Long-term quality of life for surgical and nonsurgical treatment of head and neck cancer. Arch Otolaryngol Head Neck Surg 2005;131:879-885.

87. Terrell JE, Ronis DL, Fowler KE, et al. Clinical predictors of quality of life in head and neck cancer patients. Arch Otolaryngol Head Neck Surg 2004;130:401-408.

88. Richard JM, Sancho-Garnier H, Pessey JJ, et al. Randomized trial of induction chemotherapy in larynx carcinoma. Oral Oncol 1998;34:224-228.
89. Yom SS, Machtay M, Biel MA, et al. Survival impact of planned restaging and early surgical salvage following definitive chemoradiation for locally advanced squamous cell carcinomas of the oropharynx and hypopharynx. Am J Clin Oncol 2005;28:385392.

90. Andry G, Hamoir M, Leemans CR. The evolving role of surgery in the management of head and neck tumors. Curr Opin Oncol 2005; 17:241-248.

91. Ferlito A, Corry J, Silver CE, Shaha AR, Robbins KT, Rinaldo A. Planned neck dissection for patients with complete response to chemoradiotherapy: a concept approaching obsolescence. Head Neck 2010;32:253-261.

92. Clark JR, de Almeida J, Gilbert R, et al. Primary and salvage (hypo)pharyngectomy: analysis and outcome. Head Neck 2006;28:671-677.

93. Relic A, Scheich M, Stapf J, et al. Salvage surgery after induction chemotherapy with paclitaxel/cisplatin and primary radiotherapy for advanced laryngeal and hypopharyngeal carcinomas. Eur Arch Otorhinolaryngol 2009;266:1799-1805.

94. Tsou YA, Hua CH, Lin MH, Tseng HC, Tsai MH, Shaha A. Comparison of pharyngocutaneous fistula between patients followed by primary laryngopharyngectomy and salvage laryngopharyngectomy for advanced hypopharyngeal cancer. Head Neck 2010 Feb 24. [Epub ahead of print]

95. Varghese BT, Sebastian P, Mathew A. Treatment outcome in patients undergoing surgery for carcinoma larynx and hypopharynx: a follow-up study. Acta Otolaryngol 2009;129:1480-1485.

96. Dirven $\mathrm{R}$, Swinson BD, Gao K, Clark JR. The assessment of pharyngocutaneous fistula rate in patients treated primarily with definitive radiotherapy followed by salvage surgery of the larynx and hypopharynx. Laryngoscope 2009;119:1691-1695.

97. Homma A, Sakashita T, Oridate N, et al. Importance of comorbidity in hypopharyngeal cancer. Head Neck 2010;32:148-153.

98. Gourin CG, Johnson JT. A contemporary review of indications for primary surgical care of patients with squamous cell carcinoma of the head and neck. Laryngoscope 2009;119:2124-2134.

99. Wong RJ, Shah JP. The role of the head and neck surgeon in contemporary multidisciplinary treatment programs for advanced head and neck cancer. Curr Opin Otolaryngol Head Neck Surg 2010;18:79-82.

100. Chen SW, Yang SN, Liang JA, Lin FJ, Tsai MH. Prognostic impact of tumor volume in patients with stage III-IVA hypopharyngeal cancer without bulky lymph nodes treated with definitive concurrent chemoradiotherapy. Head Neck 2009; 31:709--716.

101. Takes RP, Rinaldo A, Silver CE, et al. Future of the TNM classification and staging system in head and neck cancer. Head Neck $2010 \mathrm{Feb} 26$. [Epub ahead of print] 